\title{
A Reflection of Nature Philosophy in Architecture with a Metaphysical Perspective
}

\author{
Matineh Bakhtiari \\ Architecture Expert, University of nonprofit Rassam, Karaj, Alborz, Iran \\ Nadia Zahabi \\ Architecture Expert, Islamic Azad University of Hashtgerd, Tehran, Iran
}

Mohammad Behzadpour

Assistant Professor and Faculty Member, Department of Architecture, Faculty of Engineering, Hashtgerd Branch, Islamic Azad University, Hashtgerd, Alborz,Iran

\begin{abstract}
This paper indicates that the absence of nature as a great source of energy causes daily tensions in architecture and the fabric of life itself. It directly implies that this loss of contact with the source causes abnormalities in society. The discovery of metaphysical forces of nature and their impacts on human souls opened a chapter for such energies to be used in quotidian life. Considering the relation between human and nature from one side and the architecture and nature's metaphysics interaction, this research was after finding the righteous application of such elements in architecture and through that in society.
\end{abstract}

Conclusions and findings are represented on relation between human soul and nature.

Keywords: Tension, Metaphysics, Nature, Architecture

DOI: $10.7176 / \mathrm{ADS} / 87-04$

Publication date: November $30^{\text {th }} 2020$

\section{Introduction}

Nature has always played different roles in architecture. The role of landscapes in nature can be mentioned as an example and man has strived to match his living environment to the landscape that embodies it. In many occasions, the impact of climate on Persian architecture has been witnessed which has played a significant role in reducing the harshness and monotony of the structure. Nature affects environment in such a way that embodies its residents in a calm and soothing setting. It's also important to bear in mind the psychological impact of nature which leads us to contemplate and identify the hidden energies of nature and explore the role of metaphysics along these lines.

Modern psychologists have found that emotional, psychological and behavioral reactions of an individual along with his mental and emotional growth have close correlations with his living environment. On the other hand the harms caused by the tensions and psychological breakdowns can have significant physical, mental and social consequences. Such factors have led us to come up with appropriate plans as to how to revise our ideas of nature so we can integrate humans' life with his surrounding natural environment and by utilizing its metaphysical aspects we can guarantee the mental health of our societies.

It appears that the natural elements surrounding the architectural spaces can function as a setting which brings people together and also lead to better social interactions among the users of a complex and can result in joy and liveliness in individuals. As a result, utilizing the energies present in nature can greatly help us design suitable architectural settings.

\section{Statement of Problem}

Due to the tensions, stresses, chaos and complexities of social life in the past decades and also the fact that humans have to spent most of their time in cubic environments, it is necessary to create peaceful environments. Since we spend most of our lifetime in such architectural environments, the peace of mind that we look for should be attained from the very same architectural spaces and we have to find appropriate solutions for this problem.

The necessities of modern life such as rarity or lack of natural elements, decline in emotional relationships and other social issues stress the importance of paying more attention to architecture with natural elements so it can provide us with a tranquil environment suitable for exchange of metaphysical energies. We can also state that nature can serve as a factor leading to social dynamics, culture and mental health in individuals. In order to achieve peace of mind in today's societies, we have to return to our roots in nature so we can live in harmony and balance. Due to the harms inflicted upon the earth by humans, we can confidently state that one of the main reasons for the current tensions and issues in the world is destroying nature.

The role of metaphysics in ancient architecture is a palpable one and it can easily be noticed. As an example 
of such metaphysical impacts, the element of small pools in traditional Persian houses would make the air tender and also lead to peace of mind in individuals. Equipped with the knowledge of humans state of mind and psychology, Persian architects used to design interior spaces without considering any personal benefits in the process and they also honored beliefs, traditions and religious values which all play a key role in our mental health. As a result, anyone stepping in such places which were designed in harmony with nature and human's soul would feel calm and secure.

The eyes, deemed to be the windows to our souls, would recognize these images as in line with the soul and nature of oneself and then would send them to the brain, the humans' processing center. With satisfaction and without any resistance, brain would then receive the gist of the massage and transfer the feeling to other organs in the body. Naturally, upon sensing the feeling, a positive energy would be generated which would lead to calmness and peace of mind in the individual so they can be more organized, calm and better-tempered. That is the reason why the elderly in our society who have grown up in such environments are today a shining example of being a human with excellent personality traits including calmness, patience, and generosity and also committed to moral principles (Nayebi et al, 2007).

Our ancestors used to live in traditional houses with open and large yards with neat gardens filled with plants and flowers and tall trees. As a result, such environments would bring people together. Such settings did not only add to aesthetic aspects of the structure but also, from a metaphysical perspective, it would lead to an appropriate distribution of positive energy in the atmosphere of the house, itself an important factor in creating a soothing environment in that era. Unfortunately, such natural designs have today been replaced with towers and skyscrapers. Of course, nowadays houses in cities have yards but not for the purpose of embracing natural elements, but they solely serve as parking spaces for cars. Today's world regards itself as a separate entity from nature and as a result architecture of houses and apartments has increasingly drifted away from nature and also human nature. Today's houses and apartment buildings lack any connections to human nature and his characteristics. As a result, in order to bring back the true definition of housing, we ought to make peace with the ideas and solutions of the past. Embracing natural elements in the modern architecture can potentially make up for the harms that humans have inflicted upon nature and allow us to reconsider our past mistakes and approaches so we can once again make peace with nature (Mehdinejad et al, 2012).

Today, contemporary architecture has basically ignored the theory of harmony with nature for a variety of reasons including a quantitative and profit-oriented approach to lands and house building, inattention to spatial quality and meaning, and ignoring the effects of energies of nature on human mind. One of the main roles of nature is to create a setting suitable for social interactions and gatherings. Relations and interactions are some of the necessities of any environment suitable for humans to live in.

As the saying goes "a healthy mind in healthy body". In order to have a healthy body and mind, we should have a certain degree of peace of mind. We already know that a suitable natural environment can have a huge positive effect on the human mind. Today's depression and diseases are closely linked to the damage we cause to the nature. There is no doubt that the destruction of nature can have a negative impact on humans' demeanor, and it can have destructive consequences for individuals and the society.

We can then conclude that modern architecture has adopted an arrogant approach and it has destroyed almost all the natural elements in the environment by stealing the entire spotlight to itself. However, in the past, nature was considered as an actual part of architecture. On the other hand, the modern architecture is lacking in natural elements and its energy. Such form of architecture, without any cultural, historical and social roots, continues to exist today and this has exacerbated into identity crisis, stress and tensions in settlements and their occupants. These issues have led us to conduct this study.

In order to revive such tranquility, it is necessary to bring back nature and the concept of metaphysics into architecture so we can enhance and improve today's architecture. Just the same way that wall is an inseparable part of architecture nature has to have the same importance.

We have to take into consideration that human beings are inseparable parts of nature and these two have a close relationship. Islam has also recognized such an important connection and stressed its importance which is also the subject of the present study. The research method used for this study is descriptive analysis and qualitative.

\section{Theoretical basics}

Cosmic energy is a material that cannot be seen with our eyes but exists throughout the universe and it embodies all the angles of the known universe. It can be observed through physical science; it is internal and not palpable.

Once we split a particle, we can observe a movement which is responsible for turning the internal particles into motion; this phenomenon is known as cosmic energy. There are a variety of energies present in our surrounding environment but since there is not much information regarding them, not everyone can utilize them. As a result, metaphysics can be termed as metaphysical and supernatural phenomenon related to human dimensions. The whole universe is composed of this energy. The creator who is responsible for the movement of 
the entire universe is more powerful than cosmic energy and he is capable of controlling all the energy in the universe.

Humans as the most prominent being in the universe can have all the existing energies stored within them and utilize kinetics and potential energies in the universe. Certainly, humans are a form of energy living in an environment filled with cosmic energies. All the beings and objects can affect one another in this setting and by utilizing positive aspects of metaphysics we can increase the energies in individuals.

The existence of this energy is clear to everyone. As an example, we have often come across people who we have described as having positive or negative vibes or energies. Such descriptions are implicit references to the same concept of metaphysics which has embodied all the individuals. In case we don't find a way to discharge negative energies or attract positive ones, metaphysics will directly or indirectly have a negative impact on our societies in general. Metaphysics is in direct relationship with our minds and souls and has the potential to have significant effects on our thoughts (Zinivand, 2008).

An important issue which has been consigned to oblivion today is the dimensions of human mind. You most probably have come across these situations where you despised a certain area or location or on the other hand some settings have led you to feel so calm and secure that you desired to stay there as long as possible. There is no specific definition for such emotions and we generally define them by the feelings and emotions that such areas evoke within us. This issue stems from environmental factors which we define by their positive and negative energies. Adjustment of these energies can lead to a positive atmosphere and not doing so can have a negative and repelling effect in the area. As a result we can control the distribution of energies in the environment by designing suitable houses and apartment buildings.

As the concluding remarks regarding the statements above, objects have the capability to attract and store our energies in the environment. In case we are in a good mood for a certain period of time, all our energy will be stored in the surrounding objects and the energy level of the area reaches a level on par with ours and vice versa. That's the reason why we get bored with our living environment and life feels monotonous. The solution is that you should visit some religious or natural locations so that your energy level reaches above that of your living environment.

The main connection between humans and nature is based on an internal concept known as our nature. One of the most important roles of nature is to provide a setting for social gatherings and interaction between people. Human interactions are a necessity for any suitable environment and the element of nature paves the way to achieve this purpose. However, modern architecture is lacking in such natural elements. As a result, architects not only should take into consideration the aesthetic and environmental_aspects of their designs but also should bear in mind the need to meet the occupants' needs through the energies in nature (Zehbi et al, 2016).

\section{Nature and Humans}

Human is the product of nature. Despite this fact, he rebels against nature since he believes that nature won't embrace his presence. This can be due to the existence of a form of energy present in nature which drives us to rebellion. However, this energy is nothing but a small phase of the same central energy which drives us forcefully towards love. According to a view in Chinese philosophy regarding dialectic quality of social life interactions, there is a general relationship between man and nature in which man is under the influence of nature. The harmonious relationship between man and nature is the result of what he has learned and that he has eventually acquired the ability to live in harmony with nature. On the other hand, traditional western philosophy believes that although man is under the influence of nature, but he reacts to nature and has found a way to bring elements of nature under his control through the power of intelligence and technology.

Nature can serve as a mirror with which we can rediscover ourselves. As a result, protecting nature means protecting ourselves. As many psychologists have stressed, man's relationship with nature can have very positive results for him and severing such a connection can prevent us from benefiting from many of its positive aspects. An increase in figures related to depression in big cities is directly correlated with man's separation from nature. The destruction of nature equals harming human beings, a destruction with far-reaching consequences. The same way that harming other humans is considered a punishable act in a court of law, harming nature is also an unforgivable crime. Although this crime might not appear punishable, but the negative consequences will eventually hurt humans.

Regarding the relationship between man and nature, Andro Bernan believes that since we are part of nature we have to stay in contact with it so we can be complemented. According to him, our identity is closely related to nature and he considers it as the beginning step towards environmental ethics and believes that ethics and aesthetics similar to the truth and beauty are related concepts. He then adds that it is not rational to identify our direct benefits from being in contact with nature but not do anything in that regard and that is what we are doing today )Bell, 2007).

Jung states that for him the world or nature is the same as God for others. It is very wrong to think of nature as our enemy and something to overcome. We have to think of nature as our mother who we should peacefully 
surrender to. If we take this issue into consideration we can return to our roots in the universe the same way other creatures do. All the plants, animals and humans are in fact inseparable parts of this universe (Serrano, 1968).

Throughout the history of human civilization, people of Sumer, Egypt, Greece, Iran as some of the oldest civilizations in human history and also Latin American religions were considered as advanced civilizations. Even today we believe that these ancient civilizations formed their own structure just like Teutonic and Shaman schools of thought. Even now, besides modern civilizations we can still find primitive tribes, who worship nature and animals and respect and pursue alignment with nature. Even today, Indian Americans have not departed their culture and we can use it as a lesson to learn since we consider ourselves as modern civilizations but we are far from nature.

To an Indian American, wilderness acted like a huge church in which he lived and prayed. His desperate attempts against white people were not only for the purpose of protecting his living environment but also for preserving a holy place (Nasr, 1999). In Indian mysticism, the individual learns how to become familiar with mysteries in nature and be absorbed in it so he can use its forces to his advantage. According to the faith of Mana, all the objects are sources of energy; Animism and natural manifestations are fundamental aspects of their belief system. Indian faith is deemed a subset of American faiths and it is mainly found in South America, particularly Mexico.

We can witness a form of devotion to nature and perceived importance of its metaphysical aspects in religions such as Taoism and new Confucianism. The same form of respect towards nature along with a strong sense of symbolism and a form of awareness of clarity and transparency of universe from a metaphysical perspective of the truth can also be found in Japan (Nasr, 1999).

Today we have discovered that nature used to be respected in the Shaman faith. Quotes such as" when the spring comes along, take your steps carefully for the earth is fertile" confirm the notion above. Today, during the era of destruction of earth, pollution, global warming, floods and droughts, we have come to the conclusion that their beliefs and ideas were right (Zehbi et al, 2016).

We know that Persian-Islamic architecture embodies varying aspects of capturing and utilizing energy with the purpose of balance and tranquility for its end users. Persian architecture has always sought to utilize a variety of elements such as water, soil and wind which themselves have positive effects on capturing energy (Kavianipour, 2012). During this era, water takes a practical form in architecture and architects knowingly attempt to overcome nature and organize it through understanding the laws of physics, water and their symbolic connections to humans. Besides their roles as the means of purification, the small pools in mosques have added symbolic aspects such as life and death to the meaning of water. Water is supposed to purify humans physically and mentally. We can then conclude that understanding water is the same as understanding the architecture of water (Soltanzadeh, 13).

In addition, soil can also be considered as a source of energy for humans. It appears that the closer we are to soil the more energy we absorb. One of the main advantages of one-story buildings in the past was the peace of mind which could be absorbed as a result of being in the vicinity of soil. Humans wrongly believe that happiness can be achieved from other humans. However, according to religious ideology, happiness and energy can be achieved by worshipping and prostrating on the soil. Normally the floors which are closer to the ground provide us with a more degree of tranquility and peace of mind. As a result, Islamic architecture is a shining example of using natural elements to generate a calm and tranquil atmosphere (Mohammadi \& Qobadi, 2014).

\section{From Physics to Metaphysics}

During the last 10000 years, the earth did not witness any human civilization for more than 9500 years and it means that supernatural and metaphysics came first and nature and physics came second. In other words, the traditional spiritual view was superior to a materialistic view. About four centuries ago, such a trend reversed and a materialistic view took the place of the spiritual one. This view has recently gone even further and divides the metaphysical perspective of the universe into two categories of ancient and modern civilizations. Ancientoriented civilizations linked all the phenomenon to supernatural causes and looked for physical causes. They tended to rely on observation though sometimes mixed with superstitions. However, this approach did not necessarily mean its' lack of existence. Their priority was their thinking faculties (Zehbi et al, 2016).

\section{Pyramids}

According to the latest studies, based on fundamentals of modern physics, humans are a form of energy and live in a cosmic space filled with energy in which all the objects impact one another. This issue is of importance since the continuation and fulfillment of energy is the key to success and a deeper insight of secrets of nature such as more awareness of physical world and spiritual aspects of man himself. We are cognizant of gravity and magnetism but are not aware of their importance and how they actually function. Based on previous information we know that a field of energy flows within a pyramid without having an awareness of the nature of this energy. 
Along the same lines, building engineers have conducted thorough investigations as to how to design and construct buildings and have found that spherical and pyramidal buildings provide a more degree of peace of mind for their occupants. Of course this doesn't mean that the waves within these structures are different from the ones in other structures but their shapes and the manner of reflection result in a more tranquil setting for their occupants. There is no fundamental difference between the energies present in any of these structures but a general and basic principle should be born in mind these abnormal and unknown energies do not solely gather within these structures but they certainly come from different parts of the universe, hit the earth and spread throughout our world and some people can actually use them to their advantage (Mohammadi \& Qobadi, 2014).

No matter how we imagine the nature of energy and its behavior inside pyramidal structures or the goal of architects in utilizing such energies, it is a fact that these energies exist in pyramidal shapes. Once we identify how these energies function, we might then be able to establish the foundations of a new civilization. Iranian scientists were the first to discover that the shape of structures can affect our moods. In the past, Iranian scholars, who used to worship and seek self-discovery on mountains, found out that mysterious energies existed on mountains which helped them deepen their insights. As a result, they began to construct artificial mountains particularly in regions where there were no high mountains (Scholl, 1998).

The big secret of Cheops pyramid: this big secret is the special shape of the pyramid which is the sole reason behind some forms of hidden energy present in both animals and inanimate objects. The research conducted on pyramidal objects with sizes ranging from $1 \mathrm{~cm}$ to several meters has found out that the material of these pyramids whether wood, glass or cardboard does not affect the nature of energy within these structures and that the time the material in question has to stay there for achieving a specific result is reliant upon the repetition of these experiments.

The result of these experiments is as follows:

1. Different perishable foods lasted for longer periods of time within the pyramid without spoiling

2. Contaminated water is purified and their microbes killed

3. Plants grow faster

4. Rusty metals become polished again

5. Humans can achieve peace of mind

6. Humans' thinking faculties are enhanced

7. Calmness, rejuvenescence and healing effects can be achieved more easily (Pettit, 1998)

\section{Tree energies, the result of its cone-shaped form}

By taking a closer look at different parts of a tree we discover that the apparent form of the apex of trees is very similar to a pyramid and we already know that pyramids contain metaphysical energy. Regardless of all such interpretations related to metaphysical energies present in trees, we have to put this concept in human's point of view so we can utilize the said energies. The suggested solution for this issue is to dig a hole and plant the tree there so the height of the tree is reduced. This can facilitate the observation of the tree's apex and also the absorption of its energies.

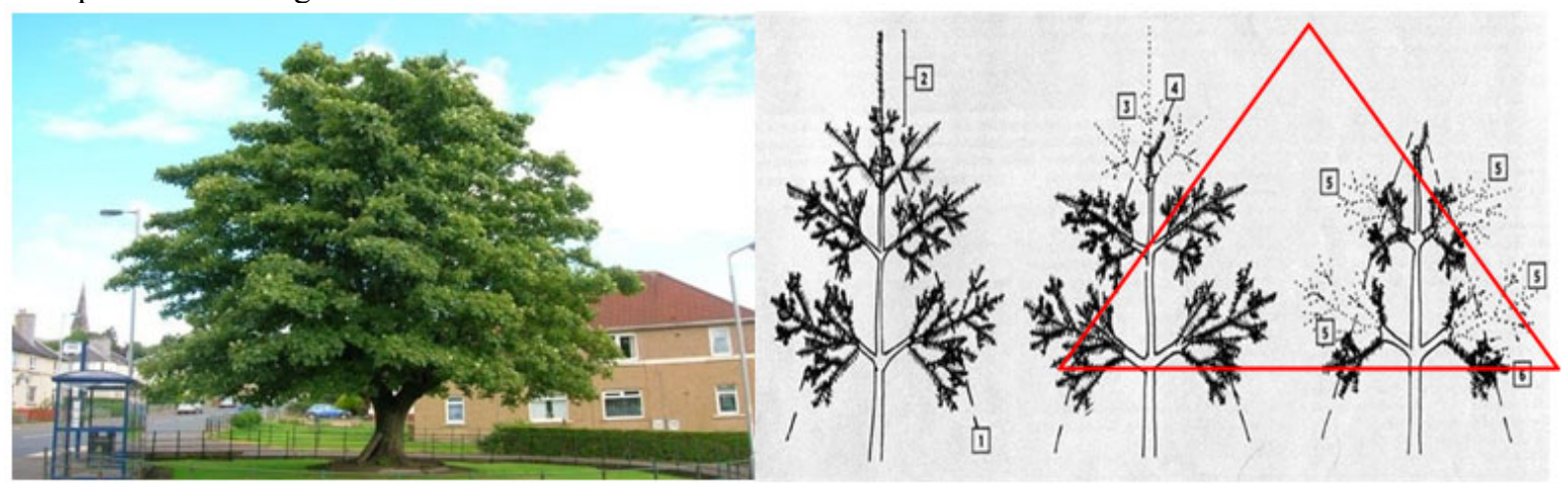

Nature and the force of love

Masters and scholars, who enjoy the love of the creator, do their jobs flawlessly and without any hubris or arrogance. They know very well that the power of love will be their main assistance. They develop this power to an extent that makes nature treats them with care and love. Poisonous snakes and wild animals that kill thousands every year treat such people with care. These people exude their inner love with such a force that wild beasts cause them no harm (Spaulding, 2014).

Asian and Eastern architectural structures have a long history of using natural materials such as wood and stressing the importance of the flow of qi, a ground energy which follows the principles of Feng Shui theory. On the contrary, western architecture stresses man's ability to use stone, brick, gravel and lime as the main materials 
in structure building. In this regard, western architecture does not share anything in common with natural elements and energies hidden in nature (Karmi \& Panahi, 2015)

Feng Shui means the art of living in harmony with nature. Feng Shui is composed of two words including Feng meaning wind and Shui meaning water. The theory of Feng Shui stems from dualism in Yin and Yang. This dualism stresses the balance of nature and also humans' contact and interaction with nature. According to this view, humans and nature complement one another, each one of them gets its value and identity from the other one and the lack of either of these two can cause a flaw in the other one (Karmi \& Panahi, 2015)._Without a natural environment, man is a being without identity. Nature is where God permeates. The same way that a mystic's lifestyle cannot be put into words, the nature of Feng Shui cannot be exactly described either. It suffices to say that Feng Shui is founded upon love. This harmony between man and universe is based on the balance of energies and this cannot be interpreted but through the concept of love.

\section{Exchange of Energies between Nature and Metaphysics:}

In general, our bodies are similar to nature and our souls can be likened to metaphysics, in that there is constant exchange of energies between the two. In order to have a better understanding of these concepts, let's take a look at some of the observations. In the endless and sophisticated world of metaphysics, wood is considered as a barrier against negative energies. In fact, by knocking on wooden objects we deflect the negative energies. Even some of the highest-ranking scholars in the field of metaphysics state that in order to deflect negative energies we should use wood. Objects such as necklaces and bracelets or the act of burning incense can drive away negative waves and energies in the environment.

In an interview with China Radio International regarding the energy of rocks, Hormuz Nabiri, the renowned lithologist states:" the majority of the people who go mountain climbing on weekends note that they can breathe easily and feel at ease when they are on a mountain. In fact, it is not just the weather in mountains that gives us such peace of mind. This is actually due to the energy released by rocks in the mountain which gives us a positive energy. If we place the energetic rock in a container with salt water and expose it to direct sunlight for as long as five hours, sunlight activates the energy hidden in the rock and salt water decontaminates the rock and removes the negative vibes. If we keep the rock in a container with purified water for some more hours, the energy of the rock enters the water and drinking such water will give you a lot of energy. In ancient civilizations, rocks and crystals used to be widely utilized for their healing effects. Medieval Shamans also used to use crystal rocks.

A simple experiment on our state of mind has concluded that natural elements affect individuals' moods. We can observe the level of people's happiness or depression in public places such as prison or home cares where they are provided with plants to water. The more lively the plant is the happier the individual and vice versa. According to previous experiences, the individuals who are more exposed to soil are less likely prone to developing Alzheimer. Elderly people can relate to soil more easily in that they feel closer to death and the higher power in the universe, God.

Nature is food for the soul and observing nature can be a rewarding act since we can understand the vastness of the creator by doing so. In other words, by asking questions we come to a better understanding of our universe. This question now might arise that why man considers what he observes as stable and constant. The reason is that we are used to a universe which appears stable and that before our birth we used to live in another universe with all the existing elements. That's why we are of the belief that everything is stable but this is not true.

Universe represents all that exits and this is not only limited to observable objects such as humans, trees, mountains etc. Universe transcends far beyond the world within our sights. Meanings and concepts are also part of this universe.

The first person to analyze the universe was Ibn Sina. A thousand years ago he described the universe through the following three concepts: Self-existent, potentially-existent and impossible to exist. Universe is the most powerful world that there is. Descartes believes that man has naturally drifted away from the source of universe and acts independently. However, he believes that man is not disconnected from the word "universe". We cannot confidently state that inexistence is the opposite side of existence. By saying so, we have caused it to exist. As a result we can state that universe is like a black hole which absorbs everything.

\section{Conclusion}

As stated earlier, the main connection between man and nature is mainly based on our own nature and origins. As a result, we can understand the true meaning of metaphysics of nature when it is categorized under our field of perception. Architecture has enabled us to come to a better understanding the concept of nature. It also helps us with utilizing nature based on a definition of "architecture-nature human". As a result, architecture can express the worldviews and beliefs held by a society in areas such as religion, nature and the power of love. Now that we have discussed some of the characteristics of energies in nature,closer attention to increasing the level of 
nsture energy used in the intital concept of etude design would allow us to use this element to distinguish green architecture from other branches.

In the end, we should bear in mind that if we believe in metaphysics in nature and consider it as the foundation of architecture, we will not restrict the values of architecture to smart responses to the end users and society or even to the appropriate use of materials and technologies or aesthetic aspects of a structure. By doing so, we will then be able to explore architectural structures from new perspectives and dimensions. Without doubt, understanding the original and foundational values of architecture is to understand its metaphysical aspects.

\section{References}

Zahabi, N. Bakhtiari, M. Behzadpour, M. (2016). Investigation of the position of nature in architecture. Sweden: Sixth International Conference on Engineering Sciences and Arts.

Naiebi, Batoul. Katebi, Fatemeh. Mehrangiz, Mazaheri. Birashk, Behrouz. (1386). The impact of interior lighting on the life quality and human moral behavior, Journal of Ethics in Science and Technology, $2^{\text {nd }}$ year, issues 3 and 4

Mehdinejad, J. \& Sirussabri, R. \& Damavandi, M. Pourasadallah, A. (1391). Architectural design with an emphasis on interaction of aesthetics and functions in nature. Journal of city identity, 10, 55-66.

Zinativand, M. (1387). Extrasensory architecture. Internal architecture magazine, 35.

Kavianipour, K. (1391). Investigation of the visual impact of environment on human behavior in detachment fold of energy.

Soltanzadeh, A. Professor at Tehran University of Medical Sciences \& Neurologist.

Mohammadi, H. Ghobadi, S. (1392) Investigation of Calmness and metaphysical energies in the pattern concepts of Islamic architecture in Iran. Tabriz: the second international congress of structures, architecture and urban development.

Scholl, B. Pettit, E. (1377). Mysterious energies in Egyptian pyramids, translated by Bashir Bakhti.

Attaei Publications.

Spaulding, B. (1388). The tomb of silence, translated by Fardieh Mahdavi Damghani. Ferdousi Publications, Eighth Edition.

Karimi, A. Panahi, S. (2015). Feng Shui. Adiban Publications.

Miguel, S. Panahi, S. (2015).Feng Shui. Adiban Publications.

Nasr, H. (1379). Man and nature, translated by Abdul Rahim Govahi. Culture Office Publications.

Bell, S. (2007). Landscape pattern of perception and process, translated by Aminzadeh. Tehran: Tehran University Publications. 